\title{
Tetrahydropalmatine inhibits lipid accumulation through AMPK signaling pathway in 3T3-L1 adipocytes
}

\author{
GUANG-CHUN PIAO, GUAN-CHENG LIU, XUE-JUN JIN, DAN JIN and HAI-DAN YUAN \\ Key Laboratory for Natural Resources of Changbai Mountain and Functional Molecules, Ministry \\ of Education, College of Pharmacy, Yanbian University, Yanji, Jilin 133002, P.R. China
}

Received February 6, 2016; Accepted February 21, 2017

DOI: $10.3892 / \mathrm{mmr} .2017 .6473$

\begin{abstract}
Tetrahydropalmatine (THP), one of the active components of Rhizoma corydalis, has been reported to exert several pharmacological effects, including anti-inflammatory, anti-tumor and analgesic activities. However, its effect on obesity and the underlying molecular mechanisms that may be involved have not yet been elucidated. In the present study, the inhibitory effects of THP on the adipogenesis in 3T3-L1 adipocytes was examined using hstology, western blotting and RT-qPCR. THP was identified to significantly suppress lipid accumulation in 3T3-L1 cells and it inhibited pre-adipocyte differentiation in a concentration-dependent manner, as evidenced by the reduced formation of lipid droplets and decreased triglyceride levels and glycerol-3-phosphate dehydrogenase activity. THP downregulated the adipogenesis-associated protein and gene expressions of sterol regulatory element-binding protein 1 , fatty acid synthase, stearoyl-CoA desaturase 1, peroxisome proliferator activated receptor $\gamma$ and CCAAT/enhancer binding protein- $\alpha$ in a concentration-dependent manner. In addition, it reduced adipocyte fatty acid binding protein and glycerol-3-phosphate acyltransferase gene expression in a concentration-dependent manner. Conversely, THP increased the mRNA expression of carnitine palmitoyltransferase 1 in a concentration-dependent manner. Furthermore, THP increased AMP-activated protein kinase (AMPK) and acetyl-CoA carboxylase phosphorylation in a concentration-dependent manner. These results suggested that anti-adipogenic activity of TPH may be mediated via the AMPK pathway in 3T3-L1 cells.
\end{abstract}

Correspondence to: Dr Hai-Dan Yuan, Key Laboratory for Natural Resources of Changbai Mountain and Functional Molecules, Ministry of Education, College of Pharmacy, Yanbian University, 977 Gongyuan Street, Yanji, Jilin 133002, P.R. China

E-mail: hdyuan@ybu.edu.cn

Key words: tetrahydropalmatine, AMP-activated protein kinase, 3T3L1 adipocyte, adipogenesis

\section{Introduction}

Obesity is a major public health problem worldwide. It is associated with increased risks of various diseases, including type 2 diabetes, hyperlipidemia, hypertension, arteriosclerosis, fatty liver and cardiovascular diseases $(1,2)$. Obesity triggers the transformation of pre-adipocytes into adipocytes, and is characterized an increase in number and size of mature adipocytes (3). Adipose tissue is primarily composed of adipocytes, and is important for energy homeostasis by regulating lipid metabolism (4). Therefore, adipocyte differentiation and the amount of lipid accumulation are involved in the expansion of adipose tissue (2).

The 3T3-L1 cell line is a well-established model for studying the conversion of pre-adipocytes into mature adipocytes and investigating the molecular mechanisms of lipogenesis and adipogenesis (5). Lipogenesis and adipogenesis are controlled by several transcription factors such as sterol regulatory element-binding protein 1 (SREBP1), peroxisome proliferator activated receptor $\gamma(\operatorname{PPAR} \gamma)$ and CCAAT/enhancer binding protein- $\alpha(\mathrm{C} / \mathrm{EBP} \alpha)$, which are primarily expressed in adipose tissue (6). These transcription factors regulate the lipid homeostasis by modulating the expression of target genes, including fatty acid synthase (FAS), stearoyl-CoA desaturase-1 (SCD1), glycerol-3-phosphate O-acyltransferase (GPAT), adipocyte protein 2 (aP2), associated with fat accumulation. Furthermore, AMP-activated protein kinase (AMPK) activation can inhibit pre-adipocyte differentiation and is accompanied by inhibition of transcription factors, including SREBP1, PPAR $\gamma$ and $\mathrm{C} / \mathrm{EBP} \alpha(7)$. AMPK activation inhibits acetyl-CoA carboxylase (ACC) activity directly via phosphorylation, and thus increases expression of carnitine palmitoyltransferase I (CPT1) (8). Therefore, AMPK signaling pathway is considered to be an important anti-obesity mechanism.

Yanhusuo (Corydalis yanhusuo W.T. Wang extract), is a well-known traditional Chinese herbal medicine, and has been used in China for treating pain, including chest impediment, heart pain, dysmenorrhea and amenorrhea (9). The pharmacological effects of yanhusuo are attributable mainly to the alkaloids in the plant, and studies have documented the hepatoprotective effects (10), anti-tumor (11), anti-inflammatory (12) and anti-hypertensive (13) activities of the extract. Tetrahydropalmatine (THP) is the main active component of yanhusuo. Recently, THP has received 
increasing attention because various pharmacological actions, including anti-coagulant, anti-nociceptive, anti-hyperalgesic, anti-oxidant, anti-viral and anti-inflammatory effects, were demonstrated to be induced by this compound (14). However, there are no reports documenting the anti-obesity effect of THP. The present study investigated the inhibitory effect of TPH on differentiation of 3T3-L1 pre-adipocytes and the mechanisms of action.

\section{Materials and methods}

Materials. THP was purchased from Sigma-Aldrich; Merck KGaA (Darmstadt, Germany). 3T3-L1 mouse embryonic fibroblast cells were purchased from the American Type Culture Collection (Manassas, VA, USA). Dulbecco's Modified Eagle's medium (DMEM), fetal bovine serum (FBS), and bovine calf serum (BCS), were purchased from Gibco; Thermo Fisher Scientific, Inc. (Waltham, MA, USA). All chemicals were purchased from Sigma-Aldrich; Merck KGaA. Rabbit anti-mouse polyclonal FAS $(3180 ; 1: 2,000)$, rabbit anti-mouse monoclonal SCD1 (2794; 1:2,000), rabbit anti-mouse polyclonal PPAR $\gamma(2435 ; 1: 2,000)$, rabbit anti-mouse polyclonal $\mathrm{C} / \mathrm{EBP} \alpha(2295 ; 1: 2,000)$, rabbit anti-mouse polyclonal phospho (p)-AMPK $\alpha(2531 \mathrm{~L} ; 1: 2,000)$, rabbit anti-mouse polyclonal AMPK $\alpha(2532 \mathrm{~S} ; 1: 2,000)$, rabbit anti-mouse polyclonal p-ACC (3661L; 1:2,000) and rabbit anti-mouse polyclonal ACC (3662; 1:2,000) antibodies were from Cell Signaling Technology, Inc. (Danvers, MA, USA). SREBP1 (sc-366; $1: 1,000)$, actin (sc-1616; 1:1,000) and horseradish peroxidase (HRP)-conjugated goat anti-mouse polyclonal IgG antibody (sc-1616; 1:1,000) were obtained from Santa Cruz Biotechnology, Inc. (Dallas, TX, USA). Reverse transcriptase was supplied by Promega Corporation (Madison, WI, USA). QuantiTect SYBR-Green PCR kit was purchased from Qiagen GmbH (Hilden, Germany).

Cell culture and adipocyte differentiation. Mouse 3T3-L1 pre-adipocytes were cultured at $37^{\circ} \mathrm{C}$ with $5 \% \mathrm{CO}_{2}$ atmosphere in DMEM medium, 10\% BCS, $100 \mathrm{U} / \mathrm{ml}$ penicillin, $100 \mu \mathrm{g} / \mathrm{ml}$ streptomycin. For standard adipocyte differentiation, 3T3-L1 cells were plated at a density of $5 \times 10^{5} \mathrm{cell} / \mathrm{ml}$ and incubated for 2 days until they reached confluence. Pre-adipocytes (designated day 0 ) were cultured in differentiation medium (DMEM, 10\% FBS, 0.5 mM 3-isobutyl-1-methylxanthine, $1 \mu \mathrm{M}$ dexamethasone and $10 \mu \mathrm{g} / \mathrm{ml}$ insulin) for 4 days, switched to post- differentiation medium containing $10 \%$ FBS and $10 \mu \mathrm{g} / \mathrm{ml}$ insulin, and then changed to $10 \%$ FBS medium for an additional 2 days. During adipocyte differentiation, 3T3-L1 cells were treated with THP at a concentration of $0,10,20$ or $40 \mu \mathrm{M}$ from day $0-4$. The positive control was treated $10 \mu \mathrm{M}$ of pioglitazone (PIO; Sigma-Aldrich; Merck KGaA) and the same concentration of differentiation standard adipogenic medium.

Cell viability assay. Cells were seeded at $5 \times 10^{3}$ cells/well in 96-well plate and incubated in the presence or absence of THP $(0,10,20$ or $40 \mu \mathrm{M})$. Following $96 \mathrm{~h}$ of culturing, $20 \mu \mathrm{l}$ of Cell Titer $96^{\circledR}$ AQueous One Solution Cell Proliferation Assay kit reagent (Promega Corporation, Madison, WI, USA) was added to each well, incubated for $1 \mathrm{~h}$, and absorbance at $490 \mathrm{~nm}$ was measured using a microplate reader.
Measurement of triglyceride (TG) content. For TG determination, cells were collected and lysed in lysis buffer (25 mM sucrose, $20 \mathrm{mM}$ Tris- $\mathrm{HCl}, 1 \mathrm{mM}$ EDTA and $1 \mathrm{mM}$ EGTA). The cellular contents of TG were measured using Infinity $^{\mathrm{TM}}$ triacyglycerol reagents (Asan Pharmaceuticals Co., Ltd., Seoul, Korea) according to the manufacturer's instructions. The protein concentration was determined by using a Bio-Rad protein assay reagent (Bio-Rad Laboratories, Inc., Hercules, CA, USA) according to the manufacturer's instructions.

Measurement of glycerol-3-phosphate dehydrogenase $(G P D H)$ activity. For GPDH determination, cells were harvested on day 8 , washed twice with PBS, and collected with a scraper into $25 \mathrm{mM}$ Tris buffer containing $1 \mathrm{mM}$ EDTA and $1 \mathrm{mM}$ dithiothreitol ( $\mathrm{pH}$ 7.5). The harvested cells were sonicated for $10 \mathrm{sec}$, and then centrifuged at $6,950 \mathrm{x} \mathrm{g}$ for $5 \mathrm{~min}$ at $4^{\circ} \mathrm{C}$. The supernatants were analyzed using GPDH activity assay kit (Takara Bio, Inc., Otsu, Japan) according to the manufacturer's protocols.

Oil-red $O$ staining. Following differentiation, culture medium was removed and cells were gently rinsed with PBS once. Cells were fixed with $10 \%$ formalin for $1 \mathrm{~h}$ at room temperature, and then stained with filtered Oil Red O solution (6:4 ratio of stock solution and water) for $2 \mathrm{~h}$ at room temperature. Finally, cells were washed with distilled water and dried. The images were captured using an Olympus IX71 inverted microscope (Olympus Corporation, Tokyo, Japan). The stained lipid droplets were dissolved in isopropanol and quantified by spectrophotometrical analysis $(540 \mathrm{~nm})$.

Western blot analysis. Cells were harvested, and total protein extracts were prepared using a PRO-PREP ${ }^{\mathrm{TM}}$ protein extraction solution (Intron Biotechnology, Inc., Seongnam, Korea) and insoluble protein was removed by centrifugation at $11,750 \mathrm{x} \mathrm{g}$ for $15 \mathrm{~min}$ at $4^{\circ} \mathrm{C}$. The supernatant was collected from the lysates and protein concentrations were determined using a Bio-Rad protein assay reagent (Bio-Rad Laboratories, Inc.) according to the manufacturer's instructions. For western blotting, $40 \mu \mathrm{g}$ protein per lane was separated on an $8 \%$ SDS-PAGE and then transferred to polyvinylidene difluoride membrane (EMD Millipore, Billerica, MA, USA). The membranes were incubated with blocking solution (tris-bufferd saline/Tween 20, TBST) containing 5\% skim milk (w/v) for $1 \mathrm{~h}$ at room temperature, followed by incubation overnight at $4^{\circ} \mathrm{C}$ with the indicated primary antibodies and further incubated with the HRP-conjugated secondary antibodies for $1 \mathrm{~h}$ at room temperature. Immunoreactive proteins were visualized by enhanced chemiluminescence solution (GE Healthcare Life Sciences, Chalfont, UK) Image J software version 1.50 (http://rsb.info.nih.gov/ij/download.html; National Institutes of Health, Bethesda, MD, USA) was used for the quantification of the results of western blotting.

RNA isolation and reverse transcription-quantitative polymerase chain reaction ( $R T-q P C R)$. Total mRNA was isolated using an Easy-Blue kit (Intron Biotechnology, Inc., Seongnam, Korea) according to the manufacturer's instructions. RNA was quantified using a Nanodrop ND-1000 UV-Vis 

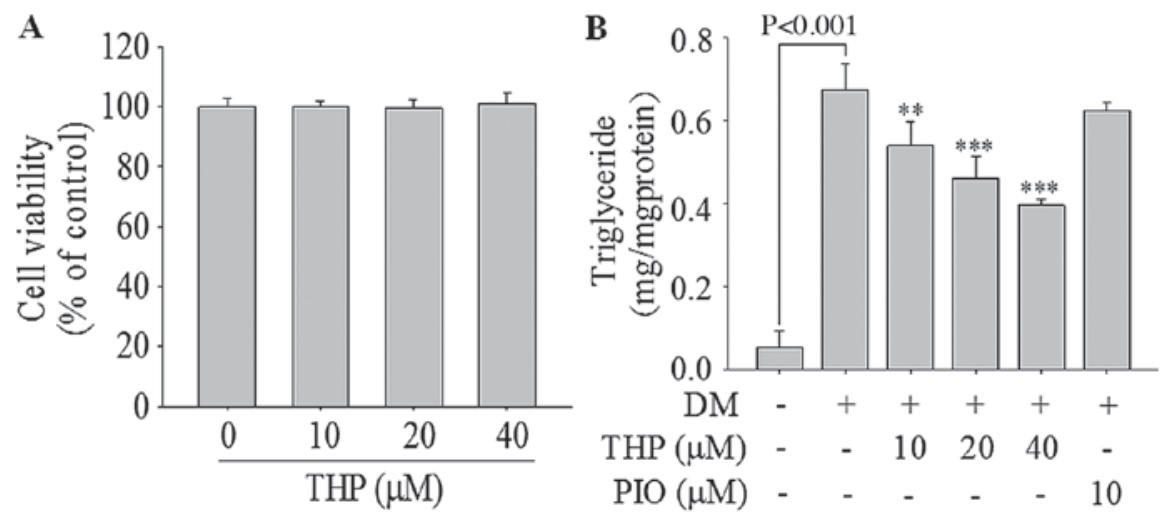

Figure 1. Effect of THP on 3T3-L1 cell viability and triglyceride contents. (A) Cells were treated with various concentrations of THP $(0,10,20$ or $40 \mu \mathrm{M})$ for $96 \mathrm{~h}$, and cell viability was measured by MTS assay. (B) 3T1-L1 cells were treated with THP at indicated concentrations and triglyceride contents were quantified. Data are represented as the mean \pm standard error of the mean $(n=3) .{ }^{* *} \mathrm{P}<0.01$ and ${ }^{* * * *} \mathrm{P}<0.001 \mathrm{vs}$. DM control. THP, tetrahydropalmatine; DM, differentiation medium; PIO, pioglitazone.

spectrophotometer (Nanodrop; Thermo Fisher Scientific, Inc., Wilmington, DE, USA). SREBP1c, FAS, SCD1, GPAT, PPAR- $\gamma, \mathrm{C} / \mathrm{EBP} \alpha$, aP2, CPT1 and $\beta$-actin mRNA levels were measured by a LightCycler Real-Time PCR system (Roche Applied Science, Penzberg, Germany) using the QuantiTect SYBR-Green PCR kit. mRNA levels were normalized to the housekeeping gene, $\beta$-actin. The primer sequences used were as follows; SREBP1c, sense 5'-GCGCTACCGGTCTTC TATCA-3', anti-sense 5'-TGCTGCCAAAAGACAAGGG-3'; FAS, sense 5'-GATCCTGGAACGAGAACAC-3', anti-sense 5'AGACTGTGGAACACGGTGGT-3'; SCD1, sense 5'-CGA GGGTTGTTGTTGATCTG-3', anti-sense 5'-ATAGCA CTGTTGGCCCTGGA-3'; GPAT, sense 5'-GGTAGTGGA TACTCTGTCGTCCA-3', anti-sense 5'-CAGCAACATCAT TCGGT-3'; PPAR $\gamma$, sense 5'-AGGCCGAGAAGGAGAAGC TGTTC-3', anti-sense 5'-TGGCCACCTCTTTGCTCTTGC TC-3'; C/EBP $\alpha$, sense 5'-GGGTGAGTTCATGGAGAA TGG-3', anti-sense 5'-CAGTTTGGCAAGAATCAGAGC A-3'; aP2, sense 5'-TCTCACCTGGCCTCTTCCTTTGGC TC-3', anti-sense 5'-TTCCATCCAGGCCTCTTCCTTTGGC TC-3'; CPT1, sense 5'-TGTCCAAGTATCTGGCAGTCG-3', anti-sense 5'-CATAGCCGTCAGCAACC-3'; $\beta$-actin sense 5'-GGACTCCTATGGTGGGTGACGAGG-3', anti-sense 5'-GGGAGAGCATAGCCCTCGTAGAT-3'. RT-qPCR was performed in a $25 \mu \mathrm{l}$ reaction mixture containing $1 \mu \mathrm{l}$ cDNA and primers using the ABI PRISM 7000 (Applied Biosystems; Thermo Fisher Scientific, Inc.). The double-stranded DNA-specific dye, SYBR-Green I, was incorporated into the PCR buffer provided by the QuantiTect SYBR-Green PCR kit (Qiagen $\mathrm{GmbH}$ ) to quantitate the PCR products by using the ${ }^{\Delta \Delta C q}$ method (15). PCR was performed at $95^{\circ} \mathrm{C}$ for $30 \mathrm{sec}$, followed by $60^{\circ} \mathrm{C}$ for $30 \mathrm{sec}$, and $72^{\circ} \mathrm{C}$ for $1 \mathrm{~min}$. The last cycle was followed by a final extension step of $72^{\circ} \mathrm{C}$ for $10 \mathrm{~min}$.

Statistical analysis. Results were represented as mean \pm standard error of the mean and differences between groups were analyzed by one-way analysis of variance followed by Student Newman Keuls. $\mathrm{P}<0.05$ was considered to indicate a statistically significant difference. Calculations were performed using the SigmaStat software version 3.5 (Systat Software, Inc., Chicago, IL, USA).

\section{Results}

Effects of THP on cell viability. To examine the cell viability following THP treatment, 3T3-L1 cells were treated with various concentrations of THP $(0-40 \mu \mathrm{M})$ for $96 \mathrm{~h}$. THP did not exhibit any cellular toxicity up to $40 \mu \mathrm{M}$ (Fig. 1A), and thus concentrations from 0 to $40 \mu \mathrm{M}$ of THP were employed in the subsequent studies. To explore the effect of THP on differentiation of pre-adipocytes into adipocytes, 3T3-L1 pre-adipocytes were treated with various concentrations of THP $(0,10,20$ or $40 \mu \mathrm{M})$ for 4 days.

Effects of THP on adipocyte differentiation. On the day 8 of incubation, the change in the contents of TG in THP-treated adipocytes was examined. As demonstrated in Fig. 1B, THP significantly reduced TG accumulation in a concentration-dependent manner. Compared with the differentiation medium control level, TG contents were significantly reduced by $13.1 \%$ at $10 \mu \mathrm{M}(\mathrm{P}<0.01), 34.4 \%$ at $20 \mu \mathrm{M}(\mathrm{P}<0.001)$ and $44.7 \%$ at $40 \mu \mathrm{M}(\mathrm{P}<0.001)$ of THP. Additionally, lipid accumulation was quantified by Oil Red O staining. As presented in Fig. 2A, THP markedly inhibited lipid droplet accumulation in a concentration-dependent manner. Consistent with results of lipid droplet accumulation, absorbance value of the eluted dyes measured at $540 \mathrm{~nm}$ were concentration-dependently decreased (Fig. 2B). Additionally, the effect of THP on the activity of GPDH was examined, as cytosolic GPDH is important role for the synthesis of TG (16). As demonstrated in Fig. 2C, THP treatment of 3 T3-L1 adipocytes resulted in a marked decrease in GPDH activity in a dose-dependent manner. These results suggested that THP efficiently inhibited adipocyte differentiation in 3T3-L1 adipocytes and may have potential anti-obesity effects.

Effects of THP on SREBPI target protein and gene expressions. To investigate the THP on the expression of genes involved in lipid metabolism, the expressions SREBP1,FAS and SCD1 genes responsible for adipogenesis were examined using western blot analysis and RT-qPCR. THP significantly inhibited the expressions of proteins (Fig. 3A) and genes (Fig. 3B) SREBP1c, FAS and SCD1 in a concentration-dependent manner, which are all 

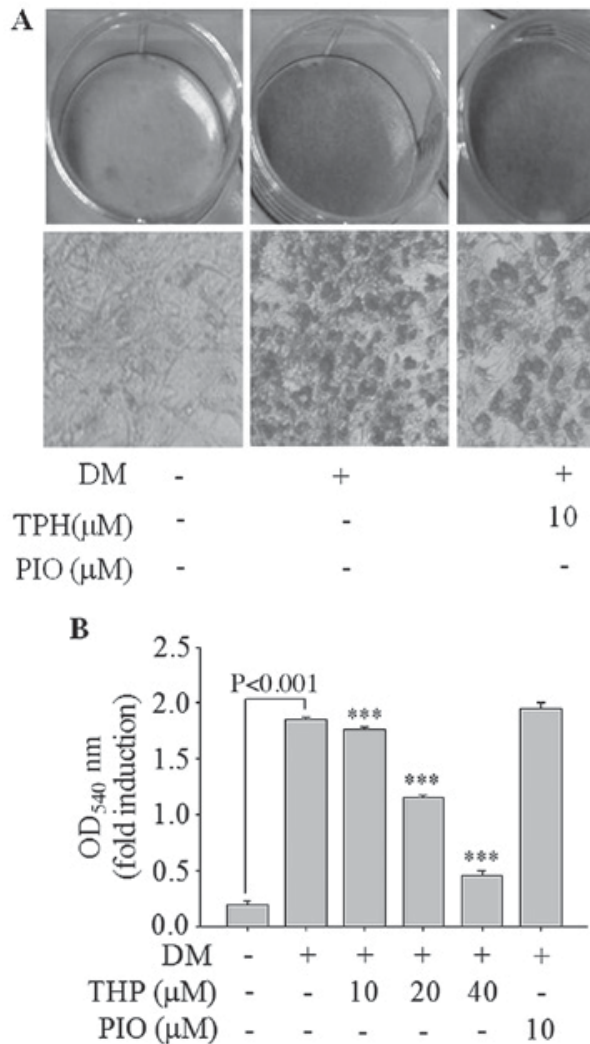
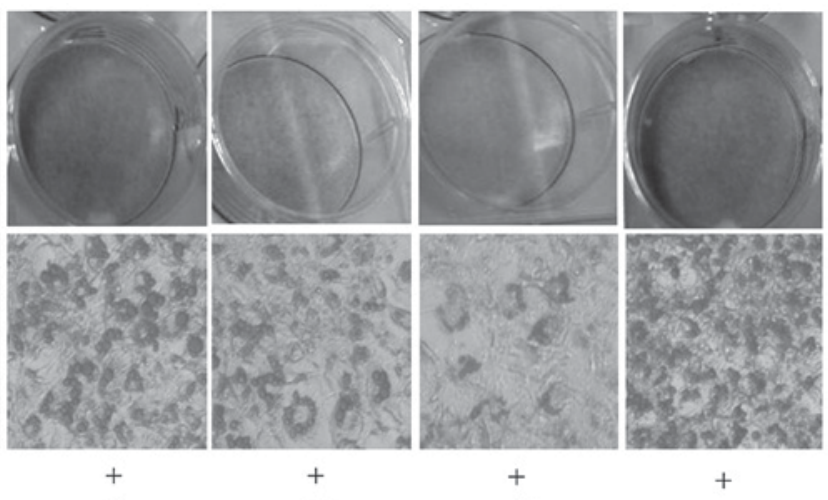

40

20

-

C

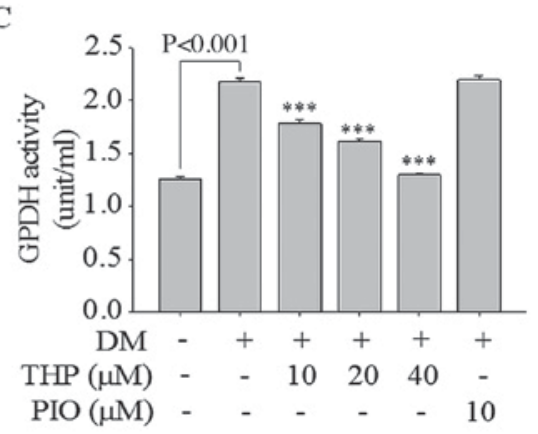

Figure 2. Effect of THP on lipid accumulation in 3T3-L1 cells. (A) Confluent cells were treated with various concentrations of THP $(0,10,20$ or $40 \mu \mathrm{M})$ from day 0 to 4. Differentiated adipocytes were stained with Oil red $\mathrm{O}$ on day 8. (B) Stained intercellular oil droplets were eluted with isopropanol and quantified by use of a spectrophotometer at $540 \mathrm{~nm}$. (C) GPDH activity was measured in 3T3-L1 cells. Data are represented as the mean \pm standard error of the mean $(\mathrm{n}=3) .{ }^{* * *} \mathrm{P}<0.001$ vs. DM control. THP, tetrahydropalmatine; GPDH, glycerol-3-phosphate dehydrogenase; DM, differentiation medium; PIO, pioglitazone.


Figure 3. Effects of THP on SREBP1 target protein and gene expressions. Confluent cells were treated with various concentrations $(0,10,20$ or $40 \mu \mathrm{M})$ of THP from day 0 to 4 . On day 8 , completely differentiated cells were lysed to extract total protein. (A) Protein extracts were prepared and subjected to western blot analysis using SREBP1c, FAS and SCD1, (B) On day 8, completely differentiated cells were used to extract total mRNA. The expression of adipogenesis-associated genes SREBP1c, FAS, SCD1 and GPAT were measured by reverse transcription-quantitative polymerase chain reaction. Data are represented as the mean \pm standard error of the mean $(n=3) .{ }^{* *} \mathrm{P}<0.01 \mathrm{vs}$. DM control. THP, tetrahydropalmatine; DM, differentiation medium; SREBP1c, sterol regulatory element-binding protein 1; FAS, fatty acid synthase; SCD1, stearoyl-CoA desaturase-1; GPAT, glycerol-3-phosphate O-acyltransferase. 

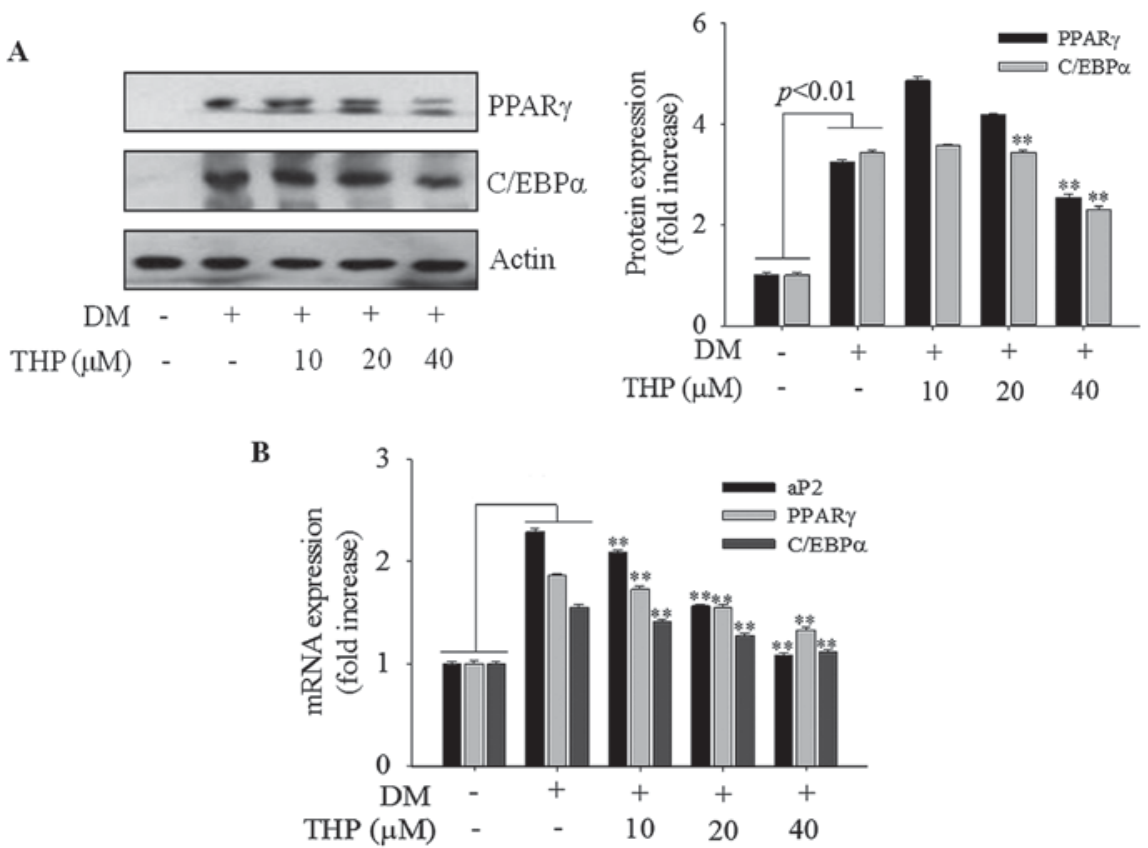

Figure 4. Effects of THP on aP2, PPAR $\gamma$ and C/EBP $\alpha$ expression. Confluent cells were treated with various concentrations $(0,10,20$ or $40 \mu \mathrm{M})$ of THP from day 0 to 4 . On day 8, completely differentiated cells were lysed to extract total protein. (A) Protein extracts were prepared and subjected to western blot analysis for PPAR $\gamma$ and CEBP $\alpha$. (B) PPAR $\gamma, \mathrm{C} / \mathrm{EBP} \alpha$ and aP2 mRNA levels were measured by reverse transcription-quantitative polymerase chain reaction. Data are represented as the mean \pm standard error of the mean $(n=3){ }^{* *} \mathrm{P}<0.01$ vs. DM control. THP, tetrahydropalmatine; DM, differentiation medium; PPAR $\gamma$, peroxisome proliferator activated receptor $\gamma ; \mathrm{C} / \mathrm{EBP} \alpha, \mathrm{CCAAT} /$ enhancer binding protein- $\alpha$; aP2, adipocyte protein 2 .

A

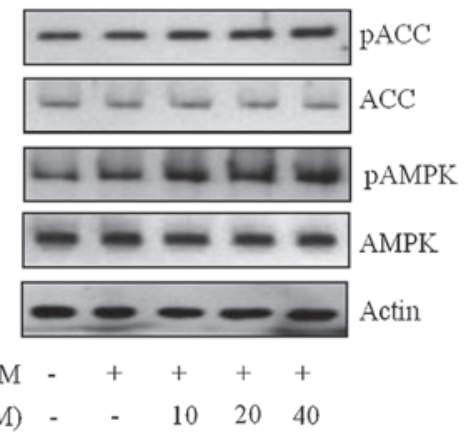

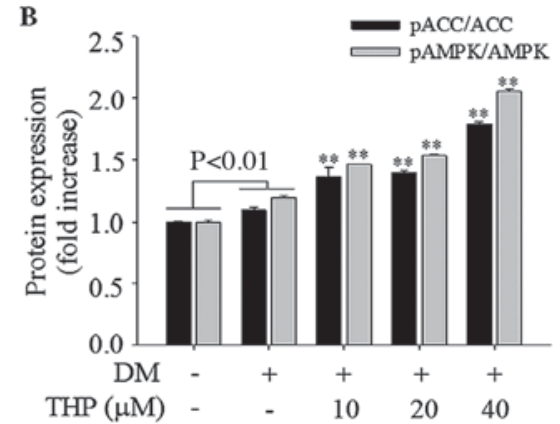

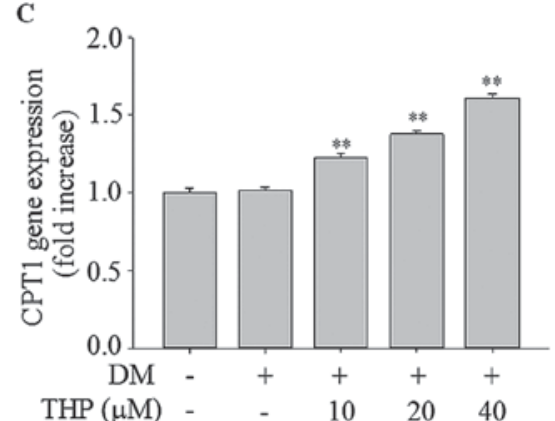

Figure 5. Effects of THP on AMPK and ACC phosphorylation and CPT1 gene expression in 3T3-L1 cells. Confluent cells were treated with various concentrations $(0,10,20$ or $40 \mu \mathrm{M})$ THP from day 0 to 4 . On day 8, completely differentiated cells were lysed to extract total protein or mRNA. (A and B) Protein extracts were prepared and subjected to western blot analysis using p-AMPK, AMPK, p-ACC, ACC, and $\beta$-actin antibodies. $\beta$-actin protein levels were used as an internal control to elevate relative expression of the protein. (C) CPT1 gene expression was measured by reverse transcription-quantitative polymerase chain reaction. Data are represented as the mean \pm standard deviation $(\mathrm{n}=3) .{ }^{* *} \mathrm{P}<0.01 \mathrm{vs}$. DM control. THP, tetrahydropalmatine; DM, differentiation medium; p, phospho; AMPK, AMP-activated protein kinase; ACC, acetyl-CoA carboxylase; CPT1, carnitine palmitoyltransferase 1.

associated with adipogenesis. In addition, the gene expression of GPAT was inhibited in a concentration-dependent manner compared with the differentiation medium group $(\mathrm{P}<0.01$; Fig. 3B). These results suggested that THP suppresses the adipogenesis gene expression of the SREBP1c target genes, such as FAS, SCD1 and GPAT, resulting in the inhibition of 3T3-L1 differentiation.

Effects of THP on PPAR $\gamma$ and C/EBP $\alpha$ expression. PPAR $\gamma$ and $\mathrm{C} / \mathrm{EBP} \alpha$ are the primary transcription factors involved in adipogenesis and lipogenesis (4). To further investigate the mechanism on 3T3-L1 differentiation, expression of PPAR $\gamma$ and $\mathrm{C} / \mathrm{EBP}-\alpha$ at the protein and mRNA level were monitored by western blot and RT-qPCR analyses, respectively. THP markedly suppressed expression of PPAR $\gamma$ and $\mathrm{C} / \mathrm{EBP}-\alpha$ protein (Fig. 4A) and mRNA (Fig. 4B) levels in a concentration-dependent manner. PPAR $\gamma$ and C/EBP- $\alpha$ promote terminal differentiation by trans-activating the expression of downstream adipocyte-specific genes, including aP2 (17). In the current results, aP2 gene expression was reduced in a concentration-dependent manner compared with the differentiation medium group $(\mathrm{P}<0.01$; Fig. 4B). These results strongly indicated that THP suppresses the expression of PPAR $\gamma$, $\mathrm{C} / \mathrm{EBP}-\alpha$ and aP2, resulting in the inhibition of 3T3-L1 differentiation.

Effects of THP on AMPK activation. To investigate whether the activation of AMPK is involved in the adipocyte TG 
lowering effect of THP, the phosphorylated forms of AMPK $\alpha$ $\left(\mathrm{Thr}^{172}\right)$, and its immediate substrate $\mathrm{ACC}\left(\operatorname{Ser}^{79}\right)$ were detected using western blotting. As demonstrated in Fig. 5A and B, THP treatment markedly enhanced the level of p-AMPK $\alpha$ $\operatorname{Thr}^{172}(\mathrm{P}<0.01)$ and $\mathrm{p}-\mathrm{ACC} \operatorname{Ser}^{79}(\mathrm{P}<0.01)$, indicating that AMPK/ACC signaling is involved in the suppression of adipogenesis caused by THP (Fig. 5A and B). AMPK leads to fatty acid $\beta$-oxidation through inactivation of ACC and increasing the expression of CPT-1 (8). The present results indicated that THP increased gene expression of CPT1 in a concentration-dependent manner $(\mathrm{P}<0.01$; Fig. 5C) compared with the control group. These results indicated that THP may inhibit adipogenesis via AMPK activation.

\section{Discussion}

Yanhusuo (Corydalis yanhusuo W.T. Wang extract) has been used as a traditional herbal medicine for centuries. Tertiary and quaternary alkaloids have been isolated from yanhusuo and identified, which are responsible for the biological activities of the crude drug (18). Among the primary constituents, THP is of particular interest. Although THP has been demonstrated to exert several pharmacological effects, including anti-coagulant, anti-nociceptive, anti-hyperalgesic, anti-oxidant, anti-viral and anti-inflammatory activities (14), its anti-obesity effect has not yet been reported. To the best of our knowledge, the present study is the first to demonstrate that THP suppressed 3T3-L1 adipocyte differentiation and lipid accumulation, and may act via the AMPK signaling pathway.

Previous studies suggest that lipid accumulation and adipocyte differentiation are associated with the occurrence and development of obesity (19-21). In the present study, THP effectively inhibited adipocyte differentiation and lipid droplet formation in a concentration-dependent manner. Additionally, THP significantly reduced TG accumulation and GPDH activity in a concentration-dependent manner, which indicates that THP inhibits adipogenesis during adipocyte differentiation. Adipocyte differentiation is the result of a complex process regulated by a number of lipogenic and adipogenic factors. SREBP1c preferentially regulates the lipogenic process by activating genes involved in fatty acid and TG (20). It is a major transcription factor involved in lipogenesis, and it induces the expression of lipogenic genes, including FAS, SCD1 and GPAT (1). Furthermore, Farmer (21) reported that SREBP1c acts to induce the expression of PPAR $\gamma$ and C/EBP $\alpha$. PPAR $\gamma$ and $\mathrm{C} / \mathrm{EBP} \alpha$ are the most important transcriptional regulators known to have central role in the adipogenesis $(6,22)$. The results of the present study demonstrated that THP downregulated the expression of SREBP1c and its target genes, FAS, SCD1 and GPAT, in a concentration-dependent manner. In addition, THP concentration-dependently downregulated the expression of PPAR $\gamma$ and $\mathrm{C} / \mathrm{EBP} \alpha$, master transcription factors involved in regulation of adipogenic genes expression, and aP2, a marker of adipocyte differentiation. These results suggested that THP exerted a lipid-lowering effect by suppressing the expression of lipogenic and adipogenic regulators that are crucial for adipocyte differentiation and lipid accumulation.

AMPK is a pivotal enzyme with a crucial role in fatty acid synthesis and oxidation via regulation of the expression and activation of enzymes and genes involved in lipid metabolism (23).
In addition, AMPK regulates pre-adipocyte differentiation and adipogenesis (24). AMPK activation directly inhibits ACC activity via phosphorylation, and indirectly inhibits ACC expression by suppressing SREBP1 $(19,25)$. Furthermore, inhibition of ACC by AMPK through phosphorylation leads to the transportation of CPT1 long chain fatty acids into the mitochondria for $\beta$-oxidation, which functions as a major regulatory enzyme in fatty acid oxidation (26).

The current results indicated that THP stimulated AMPK and ACC phosphorylation, and increased gene expression of CPT1 in a concentration-dependent manner. These results suggest that THP suppressed adipocyte differentiation through AMPK activation and ACC inactivation, and thus, inhibited the gene expression of SREBP1c, FAS, SCD1, GPAT, PPAR $\gamma$, $\mathrm{C} / \mathrm{EBP} \alpha$ and $\mathrm{aP} 2$ resulting in lipid accumulation.

In conclusion, these results suggest that THP may inhibit adipocyte differentiation and adipogenesis through the AMPK signal pathway in 3T3-L1 adipocytes. These results provide molecular information for further investigation of the mechanisms by which THP alters lipid metabolism.

\section{References}

1. Huang B, Yuan HD, Kim DY, Quan HY and Chung SH: Cinnamaldehyde prevents adipocyte differentiation and adipogenesis via regulation of peroxisome proliferator-activated receptor- $\gamma(\mathrm{PPAR} \gamma)$ and AMP-activated protein kinase (AMPK) pathways. J Agric Food Chem 59: 3666-3673, 2011.

2. Yuan HD and Piao GC: An active part of Artemisia sacrorum Ledeb. Inhibits adipogenesis via the AMPK signaling pathway in 3T3-L1 adipocytes. Int J Mol Med 27: 531-536, 2011.

3. Kowalska K, Olejnik A, Rychlik J and Grajek W: Cranberries (Oxycoccus quadripetalus) inhibit lipid metabolism and modulate leptin and adiponectin secretion in 3T3-L1 adipocytes. Food Chem 185: 383-388, 2015

4. Kim BH, Han S, Lee H, Park CH, Chung YM, Shin K, Lee HG and Ye SK: Metformin enhances the anti-adipogenic effects of atorvastatin via modulation of STAT3 and TGF- $\beta / \mathrm{Smad} 3$ signaling. Biochem Biophys Res Commun 456: 173-178, 2015.

5. Kang SI, Shin HS and Kim SJ: Sinensetin enhances adipogenesis and lipolysis by increasing cyclic adenosine monophosphate levels in 3T3-L1 adipocytes. Biol Pharm Bull 38: 552-558, 2015.

6. Ji S, Doumit ME and Hill RA: Regulation of Adipogenesis and key adipogenic gene expression by 1,25 -Dihydroxyvitamin $\mathrm{D}$ in 3T3-L1 Cells. PLoS One 10: e0126142, 2015.

7. Kang SW, Kang SI, Shin HS, Yoon SA, Kim JH, Ko HC and Kim SJ: Sasa quelpaertensis Nakai extract and its constituent p-coumaric acid inhibit adipogenesis in 3T3-L1 cells through activation of the AMPK pathway. Food Chem Toxicol 59: 380-385, 2013.

8. Ceddia RB: The role of AMP-activated protein kinase in regulating white adipose tissue metabolism. Mol Cell Endocrinol 366: 194-203. 2013

9. Wu H, Waldbauer K, Tang L, Xie L, McKinnon R, Zehl M, Yang $\mathrm{H}, \mathrm{Xu} \mathrm{H}$ and Kopp B: Influence of vinegar and wine processing on the alkaloid content and composition of the traditional Chinese medicine Corydalis Rhizoma (Yanhusuo). Molecules 19: 11487-11504, 2014.

10. Yan J, He X, Feng S, Zhai Y, Ma Y, Liang S and Jin C: Up-regulation on cytochromes $\mathrm{P} 450$ in rat mediated by total alkaloid extract from Corydalis yanhusuo. BMC Complement Altern Med 14: 306, 2014.

11. Gao JL, He TC, Li YB and Wang YT: A traditional Chinese medicine formulation consisting of Rhizoma Corydalis and Rhizoma Curcumae exerts synergistic anti-tumor activity. Oncol Rep 22: 1077-1083, 2009.

12. Yun KJ, Shin JS, Choi JH, Back NI, Chung HG and Lee KT: Quaternary alkaloid, pseudocoptisine isolated from tubers of Corydalis turtschaninovi inhibits LPS-induced nitric oxide, PGE(2), and pro-inflammatory cytokines production via the down-regulation of NF-kappaB in RAW 264.7 murine macrophage cells. Int Immunopharmacol 9: 1323-1331, 2009. 
13. Chueh FY, Hsieh MT, Chen CF and Lin MT: DLtetrahydropalmatine-produced hypotension and bradycardia in rats through the inhibition of central nervous dopaminergic mechanisms. Pharmacology 51: 237-244, 1995.

14. Lee B, Sur B, Yeom M, Shim I, Lee H and Hahm DH: L-tetrahydropalmatine ameliorates development of anxiety and depression-related symptoms induced by single prolonged stress in rats. Biomol Ther (Seoul) 22: 213-222, 2014.

15. Zhang M, Liu L, Xiao T and Guo W: Detection of the expression level of miR-140 using realtime fluorescent quantitative PCR in knee synovial fluid of osteoarthritis patients. Zhong Nan Da Xue Xue Bao Yi Xue Ban 37: 1210-1214, 2012 (In Chinese).

16. Kim YS, Lee YM, Kim JH and Kim JS: Polygonum cuspidatum inhibits pancreatic lipase activity and adipogenesis via attenuation of lipid accumulation. BMC Complement Altern Med 13: 282, 2013.

17. Choi SS, Cha BY, Lee YS, Yonezawa T, Teruya T, Nagai K and Woo JT: Magnolol enhances adipocyte differentiation and glucose uptake in 3T3-L1 cells. Life Sci 84: 908-914, 2009.

18. Wang XL, Zheng Z, Hong Z and Fan G: Advancements on chemical components and quality control of rhizoma corydalis. Lishizhen Med Mater Med Res 22: 227-229, 2011.

19. Mcilroy GD, Tammireddy SR, Maskrey BH, Grant L Doherty MK, Watson DG, Delibegović M, Whitfield PD and Mody N: Fenretinide mediated retinoic acid receptor signalling and inhibition of ceramide biosynthesis regulates adipogenesis, lipid accumulation, mitochondrial function and nutrient stress signalling in adipocytes and adipose tissue. Biochem Pharmacol 100: 86-97, 2016.
20. Rao Y, Liu H, Gao L, Yu H, Tan JH, Ou TM, Huang SL, Gu LQ, Ye JM and Huang ZS: Discovery of natural alkaloid bouchardatine as a novel inhibitor of adipogenesis/lipogenesis in 3T3-L1 adipocytes. Bioorg Med Chem 23: 4719-4727, 2015.

21. Farmer SR: Transcriptional control of adipocyte formation. Cell Metab 4: 263-273, 2006

22. Zhang X, Ji J, Yan G, Wu J, Sun X, Shen J, Jiang H and Wang H: Sildenafil promotes adipogenesis through a PKG pathway. Biochem Biophys Res Commun 396: 1054-1059, 2010.

23. Fryer LG and Carling D: AMP-activated protein kinase and the metabolic syndrome. Biochem Soc Trans 33: 362-366, 2005.

24. Kim ED, Kim E, Lee JH and Hyun CK: Gly-Ala-Gly-Val-Gly-Tyr, a novel synthetic peptide, improves glucose transport and exerts beneficial lipid metabolic effects in 3T3-L1 adipoctyes. Eur J Pharmacol 650: 479-485, 2011.

25. Zhang BB, Zhou G and Li C: AMPK: An emerging drug target for diabetes and the metabolic syndrome. Cell Metab 9: 407-416, 2009.

26. He Y, Li Y, Zhao T, Wang Y and Sun C: Ursolic acid inhibits adipogenesis in 3T3-L1 adipocytes through LKB1/AMPK pathway. PLoS One 8: e70135, 2013. 